\title{
USO DO MMPI (MINNESOTA MULTIPHASIC PERSONALITY INVENTORY) NA AVALIACÃO DE PACIENTES COM SÍNDROME DE DOR CRÔNICA
}

\author{
LI SHIH MIN * - LI LI MIN ** - LILIA FREIRE RODRIGUES DE SOUZA ***
}

\begin{abstract}
RESUMO - Utilizou-se o MMPI na avaliação de perfil da personalidade de 25 pacientes com síndrome de dor crônica. Evidenciaram-se em $64 \%$ dos pacientes alterações nas escalas 1. 2, 3. O teste mostrou-se adequado para a avaliação dos pacientes, oferecendo padronização e visualização objetiva do envolvimento psíquico. O teste pode ser útil também na avaliação do prognóstico de intervenções psicoterápicas e cirúrgicas.
\end{abstract}

Use of MM PI on the assessment of the chronic pain syndrome patients

SUMMARY - The MMPI was performed in 25 patients with chronic pain syndrome to evaluate the personality trait. The results showed an elevation in scales 1, 2,3 in $64 \%$ of the patients. The inventory was effective on the assessment of patients. It offered standardization and objective visualization of patients' psychical involvement. Also, it can serve as a prognostic factor for psychotherapy and surgery.

Definições de dor crônica podem ser confusas, dado que elas são provenientes de múltiplas especialidades médicas e direcionam a entidade, partindo de diferentes pontos de vista. Florence 3 propôs que a dor crônica é qualquer dor que persista além do que deveria ser esperado para a cura do tecido especificamente envolvido. Mas, na síndrome de dor crônica, além da dor,soma-se um conjunto de componentes funcionais ou emocionais 3. Com as implicacões psicológicas e sociais, a síndrome de dor crônica torna-se difícil ao julgamento rotineiro do clínico. Na tentativa de resolver esta deficiência, surgiram vários questionários e testes psicométricos e de personalidade i. Dentre esses foi escolhido o Minnesota Multiphasic Personality Inventory (MMPI) na avaliação da personalidade dos pacientes. O MMPI foi concebido em 1937 por Starke R. Hathaway, psicólogo, e John C. McKinley, neuropsiquiatra 5. Foi desenvolvido para auxiliar na avaliação mental de pacientes vistos na prática médica. O MMPI é considerado um dos instrumentos mais usados na «avaliação de psicopatologia» na atualidade. O inventário é constituído de 550 perguntas com respostas «certo», «errado», posteriormente convertidas em escores com o uso dos crivos de correcão. Estes escores têm como média 50 e desvio padrão 10; escores maiores que 70 ou menores que 30, i.e., variação maior que duas vezes o desvio padrão, são considerados clinicamente importantes 2,5. Atualmente os escores da escala não são mais relacionados diretamente a dado diagnóstico específico. Adota-se abordagem pela análise da configuração do traçado. São interpretados, em geral, duas ou três escalas mais elevadas, com referência a correlatos comportamentais previamente estabelecidos. Esses correlatos incluem traços de personalidade, sintomas psiquiátricos e, em última análise, diagnósticos psiquiátricos. Devido ao fato de que as escalas isoladas não são mais diagnosticas, aconselha-se referi-las pelo número em lugar do nome originais.

O presente estudo foi motivado pelas dificuldades vistas no exercício médico diário em obter-se abordagem adequada de pacientes com síndrome de dor crônica, devido às suas implicações de ordem bio-psico-social, principalmente pela deficiência na avaliação dos seus componentes funcionais ou emocionais. $O$ inventário é instrumento que facilita traçar o perfil psicológico do paciente. Não há intenção de fazer

* Médico Clínico, Hospital Regional São José; ** Residente de Medicina Interna, Hospital Universitário, Universidade de Santa Catarina (UFSC); *** Residente de Pediatria, Hospital Pequeno Príncipe, Curitiba.

Dr. Li Li Min - Disciplina de Medicina Interna, Departamento de Clínica Médica, Hospital Universitário UFSC - Campus Universitário, Trindade - 88049 Florianópolis SC - Brasil. 
distinção entre dor «orgânica», «funcional» ou «psicogênica» baseando-se no resultado do inventário.

\section{MATERIAL E MÉTODO}

Os pacientes que participaram voluntariamente deste estudo eram todos provenientes da clínica particular e foram incluídos após preencher os seguintes critérios: (1) queixa de dor com duração maior que 6 meses; ou (2) duração menor que 6 meses, mas relatando alterações do modo de ser devidas à dor; e (3) resultados de MMPI (Tabela 1) válido5. Assim, totalizaram-se 25 pacientes: 10 do sexo masculino e 15 do feminino, com idade variando de 18 a 72 anos (mediana: 48 lanos); duração da dor de 2 meses a 38 anos (mediana: 24 meses); número de médicos .iá consultados variando de 1 a 10 (mediana: 3); 4 pacientes estavam ou estiveram em tratamento' psicológico (16\%) e 1 em tratamento psicológico não por causa da dor, especificamente; 5 pacientes (20\%) utilizavam ansiolíticos e 2 (8\%), antidepressivos tricíclicos; 22 dos 25 pacientes (88\%) relatavam alteração do modo de ser pela dor e somente 3 (12\%) negavam esse tipo de alteração. Os diagnósticos informados pelos pacientes eram: cervicalgia em 8, cérvico-lombalgia em 2, lombalgia em 2, ciática em 3, lombo-ciatalgia em 1, tendinite em 2; em cada um dos 7 restantes, o diagnóstico referido era cefaléia, bursite, otalgia, coxartrose, espondilite anquilosante, lesão de menisco do joelho, lesão patelar.

Os escores encontrados foram acrescidos de fator de correção (K) para fins do estudo. Aplicou-se a análise da configuração do traçado para a interpretação, classificando os casos segundo as duas escalas clínicas mais elevadas (Tabela 2). Para as conclusões, utilizaram-se as medianas dos escores das respectivas escalas dos 25 pacientes (Tabela 3), sendo elaborado um traçado para análise (Fig. 1).

\section{RESULTADOS}

Analisando o padrão dos traçados (Tabela 2) evidenciou-se que: $40 \%$ dos pacientes apresentavam elevações nas escalas 1 e 3 e, neste grupo, 5 pacientes (20\% do total) apresentavam a clássica «Conversão em V», i.e., apresentavam escores maiores que 70 nessas escalas; $12 \%$, elevação na escala 2; $8 \%$, nas escalas 2 e 3; $4 \%$ nas escalas 1 e 2. Assim, 64\% dos pacientes apresentavam alterações nas escalas 1, 2, 3 .

\begin{tabular}{cll}
\hline Número & Sigla & Nome \\
\hline & $\mathbf{I}$ & Dúvida \\
\hline & $\mathbf{L}$ & Mentira \\
$\mathbf{F}$ & «Erro» \\
& $\mathbf{K}$ & «Correção» \\
$\mathbf{1}$ & $\mathrm{Hs}$ & Hipocondria \\
$\mathbf{2}$ & $\mathrm{D}$ & Depressão \\
$\mathbf{3}$ & $\mathrm{Hy}$ & Histeria \\
$\mathbf{4}$ & $\mathrm{Pd}$ & Desvio psicopático \\
$\mathbf{5}$ & $\mathrm{Mf}$ & Masculino-Feminino \\
$\mathbf{6}$ & $\mathrm{Pa}$ & Paranóia \\
$\mathbf{7}$ & $\mathrm{Pt}$ & Psicatenia \\
$\mathbf{8}$ & $\mathrm{Sc}$ & Esquizofrenia \\
9 & $\mathrm{Ma}$ & Hipomania \\
$\mathbf{0}$ & $\mathrm{Si}$ & Introversão social
\end{tabular}

Tabela 1 - Escalas de MMPI. As escalas não numeradas são de validade $e$ as numeradas são escalas clinicas.

\section{COMENTARIOS}

Não encontramos qualquer referência na literatura nacional a estudos em que foi aplicada a análise da configuração para comparar os resultados obtidos nesta investigação.

Para o julgamento clínico dos pacientes com síndrome de dor crônica, as escalas mais importantes são: 1 (Hs, Hipocondria), 2 (D, Depressão) e 3 (Hy, Histeria); essas escalas constituem a chamada «tríade neurótica» 5. Correlaciona-se a escala 1 a queixas vagas $\mathrm{e}$ inespecíficas da função corporal: pacientes com escores altos são interpretados como pessimistas, derrotistas em relação a perspectivas de melhora de suas vidas particulares; frequentemente são teimosos, desprovidos de ambição e, geral- 


\begin{tabular}{ccc} 
Escala & No de casos & $\%$ \\
\hline $1-3$ & 10 & 40 \\
$1-2$ & 01 & 4 \\
$1-7$ & 01 & 4 \\
$2-0$ & 03 & 12 \\
$2-3$ & 02 & 8 \\
$2-7$ & 01 & 4 \\
$3-9$ & 01 & 4 \\
$4-2$ & 01 & 4 \\
$4-3$ & 01 & 4 \\
$5-0$ & 01 & 4 \\
$5-2$ & 01 & 4 \\
$6-7$ & 01 & 4 \\
$0-1$ & 01 & 4 \\
\hline
\end{tabular}

Tabela \&- Configuraçäo dos casos pelas escalas.

\begin{tabular}{ccc}
\hline Escala & Variação & Mediana \\
\hline \hline$?$ & $50-64$ & 50 \\
I & $50-74$ & 54 \\
K & $28-80$ & 44 \\
F & $44-66$ & 50 \\
Hs & $45-89$ & 63 \\
D & $42-85$ & 59 \\
Hy & $35-78$ & 62 \\
Pd & $33-78$ & 49 \\
Mf & $27-71$ & 47 \\
Pa & $35-78$ & 47 \\
Pt & $35-73$ & 54 \\
Sc & $34-67$ & 52 \\
Ma & $31-65$ & 49 \\
Si & $37-77$ & 56 \\
\hline
\end{tabular}

Tabela 3 - Variação e mediana dos escores das escalas.

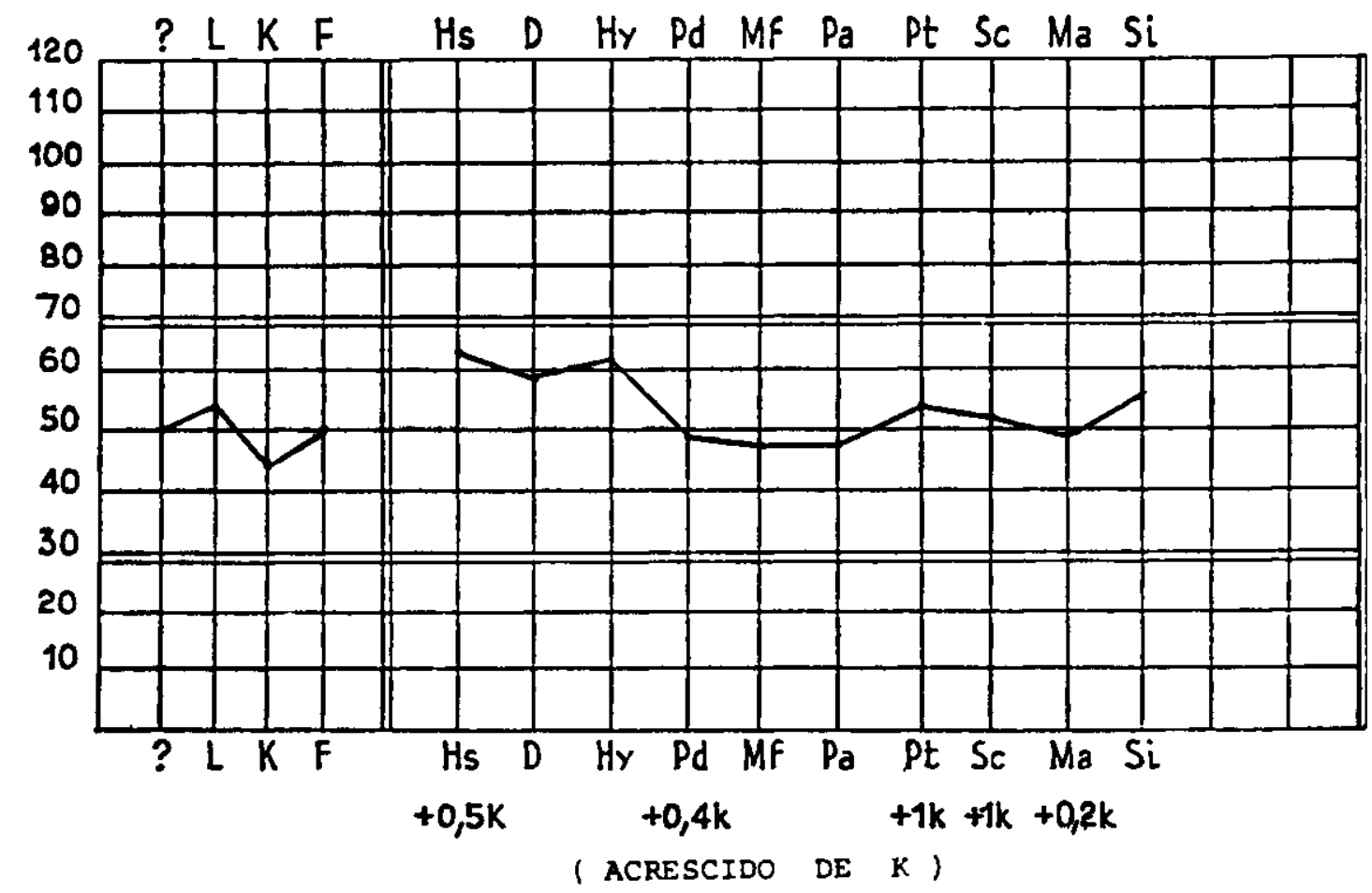

F'ig. 1 - Mediana dos escores dos 25 inventarios (MMPI). 
mente, pouco se beneficiam de intervenção psicoterápica. Quase todos os pacientes com dor lombar têm elevações nessa escala. Pela escala 2 é avaliado o grau de acometimento de depressão sintomática: desânimo, falta de esperança, insatisfação com o próprio status; ela é um dos melhores indicadores de como o paciente avalia sua imagem e seu papel no mundo; pessoas com escores altos tendem a ser silenciosas, sensíveis e de pouca sociabilidade. Pela escala 3 são explorados dois aspectos, fundamentalmente: queixas somáticas específicas e negação de qualquer dificuldade emocional ou interpessoal; pacientes com escores altos podem ser descritos como imaturos, egocêntricos; exigem atenção excessiva e são manipulativos; além disso, possuem tendência para desenvolver sintomas conversivos como um meio de resolver conflitos e evitar responsabilidades ${ }^{2}$.

A elevação nas escalas 1-3/3-1, às vezes denominada «conversão em V» ou «V psicossomático», era interpretada como indicativa de doença funcional 9. Entretanto, sua eficácia em tal sentido não foi comprovada 8.10-12. pacientes com elevação nessas duas escalas são comuns, com negação da possibilidade de qualquer tipo de conflito psicológico; tendem a ser rígidos afetivamente e podem expressar desconfortos psicológicos por queixas somáticas. Quando psicologicamente perturbados ou submetidos a stress, tendem a migrar para seu polo negativo ${ }^{4} »$ ?, somatizando seus conflitos e podendo experimentar ampla variedade de sintomas físicos. Frequentemente sentem-se ameaçados, quando confrontados diretamente à idéia de que seu mal pode ter componentes psicológicos. Intervenções cirúrgicas nesses pacientes geralmente podem acabar em maus resultados; isso frequentemente só é detectado quando várias operações já tiverem sido realizadas.

Conclusão - No traçado elaborado a partir das medianas dos escores, observamos elevação nas escalas 1,2 e 3, principalmente nas escalas 1 e 3, o que corresponde à própria definição da síndrome de dor crônica. Consideramos o MMPI um teste válido e aplicável em nosso ambiente de trabalho para compensar nossas próprias deficiências na avaliação psicológica do paciente. $O$ teste oferece as vantagens da padronização e da avaliação objetiva do psiquismo facilitando, consequentemente, a análise. Além de uma avaliação concisa e quantificada, o MMPI oferece certos indicadores para aceitação, por parte dos pacientes, da psicoterapia. Seu emprego rotineiro poderá servir como teste pré-operatório, útil no prognóstico da possibilidade de sucesso da cirurgia.

Agradecimentos - Agradecemos aos pacientes que participaram deste estudo e a Aluísio C. M. Mello por ter cedido seu equipamento de informática. Agradecimento especial é feito aos Drs. Acir Rachid e Paulo Cesar Trevisol Bittencourt.

\section{REFERÊNCIAS}

1. Blaser D. Chronic pain: a multiaxial approach to psychosocial assessment and intervention. Southern Med J 1981, 74:203-207.

2. Dennis MI>, Greene RXr, Fair S>F. The Minnesota Multiphasic Personality Inventory: general guidelines to its use and interpretation in orthopedics. Clin Orthopaed Rel Res 1980, 150-125-130.

3. Florence DW. The chronic pain syndrome. Postg Med 1981, 70:217-228.

4. Graham JR, McCord G. Interpretation of moderately elevated MMPI scores for normal subjects. J Pers Assess 1985, 49:477-484.

5. Hathaway SR, McKinley JC. Manual de MMPI. Rio de Janeiro: CEPA-Centro Editor de Psicologia Aplicada (sem data).

6. Hickey JSA, Baer PE. Abordagens psicológicas na avaliação e no tratamento da ansiedade e da depressão. Clin Med Am Norte 1988, 4:943-960.

7. Kunce JT. Normalizing the MMPI. J Clin Psychol 1976, 32:776-780.

8. Leavitt F. The value of the MMPI conversion «V» in the assessment of psychogenic pain. J Psychosom Res 1985, 29:125-131.

9. McCreary C, Turner J, Dawson E. Differences between functional versus organic low back pain patients. Pain 1977, 4:73-78.

10. Naliboff BD, Cohen MJ, Yellen AN. Does the MMPI differentiate chronic illness from chronic pain? Pain 1982, 13:333-341.

11. Rook JC, Pesch RN, Keeler EC. Chronic pain and the questionable use of the Minnesota Multiphasic Personality Inventory. Arch Phys Med Rehab 1981, 62:373-376.

12. Stone RK Jr, Rockwell FPA. Diagnosis of organic and functional pain patients with the MMPI. Psychol Reports 1983, 52:539-548. 